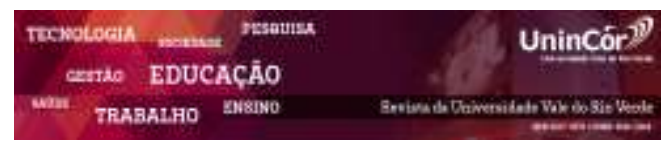

Revista da Universidade Vale do Rio Verde ISSN: 1517-0276 / EISSN: 2236-5362 Vol. 16 | n. 1 | Ano 2018

Dandara Medeiros Paiva Universidade Federal de Campina Grande-UFCG dandara.paivaa@gmail.com

Joanna Renata Santos de Almeida Silva Universidade Federal de Campina Grande-UFCG joanna.sse@hotmail.com

Joseane Xavier de Almeida União de Ensino Superior de Campina GrandeUNESC

joe_nf_ermagem@hotmail.com

Laura Raquel Araújo de Moraes Universidade Federal de Campina Grande-UFCG laura.amoraes@hotmail.com

Maria Érica Gomes da Silva Universidade Federal de Campina Grande-UFCG mariaericagomesdasilva1@gmail.com

Rosangela Vidal de Negreiros Universidade Federal de Campina Grande-UFCG rosangelavn@ufccg.edu.br

\section{ATUAÇÃO DO ENFERMEIRO NO ACOMPANHAMENTO DO USUÁRIO COM DIABETES MELLITUS: UMA VIVÊNCIA HOSPITALAR}

\section{RESUMO}

Este estudo objetiva refletir, a atuação do enfermeiro no tratamento do Diabetes Mellitus (DM) a partir da vivência em um Hospital Universitário do Interior nordestino. Trata-se de estudo empírico-descritivo, exploratório, de relato de experiência vivenciado por discentes do curso de enfermagem da Universidade Federal de Campina Grande, durante o estágio curricular Supervisionado II, com carga horária de 420 horas. A experiência ocorreu na Ala $\mathrm{C}$, com a maior predominância de pacientes com patologias endócrinas e cardíacas. O estudo foi realizado entre os meses de julho e setembro de 2016. Quanto aos resultados, ao realizar o diagnóstico situacional da Ala C, evidenciou-se a predominância de pacientes endócrinos idosos, em destaque a Diabetes Melittus, muitos com complicações em membros inferiores, que resultaram em amputações, diante de tal situação foi possível atuar e prestar cuidados que exijam uma maior habilidade técnica e raciocínio critico em pacientes com complicações. Nesse sentido, o Estágio Supervisionado II é uma etapa extremamente expressiva no processo de formação do aluno, onde proporciona ao mesmo vivenciar a rotina hospitalar por um período prolongado, como também é uma alternativa fundamental para que haja uma familiaridade maior com as atribuições do profissional de enfermagem do nível superior.

Palavras-chave: Diabetes Mellitus. Tratamento. Hiperglicemia. Pé diabético. Cuidados de enfermagem.

\section{NURSE'S ACTIVITIES IN THE USER ACCOMPANYING WITH DIABETES MELLITUS: A HOSPITAL LIVING}


extremely significant step in the student's education process, which provides the same experience the hospital routine for an extended period, it is also a key alternative so there is a greater familiarity with professional assignments of nursing top level.

Keywords: Diabetes Mellitus. Treatment. Hyperglycemia. Diabetic foot. Nursing care.

Recebido em: 10/10/2016 - Aprovado em: 10/01/2018 - Disponibilizado em: 15/07/2018

\section{INTRODUÇÃO}

O Diabetes Mellitus- DM é uma condição prevalente e de grande relevância sendo definido como um problema de saúde pública em todos os países, independentemente de seu grau de desenvolvimento. Trata-se de uma disfunção metabólica caracterizada por hiperglicemia e associadas a complicações e insuficiência de vários órgãos, especialmente olhos, rins, nervos, cérebro, coração e vasos sanguíneos. Podendo resultar de defeitos de secreção e/ou ação da insulina envolvendo processos patogênicos específicos, resistência à ação da insulina, distúrbios da secreção da insulina, entre outros (BRASIL, 2006).

Na década de 1990, o SUS passou por modificações visando substituir o atendimento hospitalar e o modelo curativo por atividades de caráter epidemiológico, com ações no campo da saúde pública, de cunho mais preventivo e coletivo, buscando a melhoria das condições de saúde. (MENICUCCI, 2014)

Com isso, ao longo do tempo, várias reformas foram implantas pelo governo com o intuito de diminuir a incidência de DM, pois a patologia apresenta elevadas proporções, com alto custo econômico e social. Porém, tem-se percebido uma maior procura aos serviços hospitalares para o controle e tratamento das complicações ocorridas pela doença.

A alteração desse quadro passa obrigatoriamente pela organização e incentivo da atenção básica, onde tem a possibilidade de implantar medidas que retardam o aparecimento da doença ou evitam seus agravos depois de instalada, diminuindo assim, o índice de internações hospitalares por conta do DM.

$\mathrm{O}$ número de diabéticos vem crescendo simultaneamente de acordo com fatores como: o aumento da obesidade e do sedentarismo, do crescimento e envelhecimento populacional, do aumento significativo da urbanização e também devido ao aumento da sobrevida de pacientes portadores de DM. Faz-se necessário quantificar a prevalência atual de diabetes com o propósito de presumir o quantitativo de diabéticos futuramente, afim de obter um planejamento cauteloso e preciso e consequentemente realizar reservas de recursos de maneira prudente $(\mathrm{SBD}$, 2014).

O DM apresenta duas formas principais, o diabetes mellitus tipo 1, que é mais agressivo, causa emagrecimento rápido, ocorre na infância e adolescência, o indivíduo não tem produção de insulina, a glicose não entra nas células e o nível 
de glicose no sangue fica aumentado. O Diabetes Mellitus tipo 2 (DM2) geralmente é diagnosticado após os quarenta anos, todavia pode ocorrer em qualquer idade. Os pacientes desse tipo do DM podem necessitar de tratamento com insulina para obter controle metabólico apropriado, entretanto esses não dependem de insulina exógena para sobreviver (SBD, 2015).

A ausência de controle glicêmico por parte dos portadores de DM acarreta em alterações fisiológicas e metabólicas que contribuem para o surgimento de complicações crônicas, entre estas podem ser citadas a cetoacidose, hiperglicemia, retinopatia, neuropatia e nefropatia. Em longo prazo, se esse controle permanecer inadequado, pode acarretar alterações mais complexas como: doença arterial coronariana e a doença cerebrovascular.

Estratégias atuais de tratamento foram desenvolvidas, bem como o estabelecimento de critérios diagnósticos da disfunção obtiveram avanços expressivos nos últimos anos. Todavia, vale ressaltar que grande parte dessas informações ainda não foram consolidadas satisfatoriamente, nesse sentido observa-se dificuldades por parte dos profissionais de saúde no que diz respeito a tomada de decisões nos pressupostos modernos da terapêutica. Nessa perspectiva faz-se necessário o avanço dos estudos atuais no intuito de que cada tratamento seja alicerçado em estudos do tipo randomizado, controlado com desfechos precisos e com amostra suficiente para responder às perguntas formuladas (SBD, 2002).

O enfermeiro por sua vez, é cabível o papel de assumir a responsabilidade de rastrear e monitorar os fatores de risco para o Pé Diabético, como também orientá-los quanto aos riscos, e, estabelecendo uma parceria com toda a equipe de saúde, determinar as intervenções básicas e planejar as atividades educativas para o autocuidado e para a manutenção de um bom controle metabólico, evitando graves complicações (TEIXEIRA, 2011)

Um plano de cuidados elaborado adequadamente e aplicado de forma correta estabelece a direção e a continuidade da assistência além de facilitar e tornar-se mais produtivo o diálogo entre os enfermeiros e a equipe multidisciplinar envolvida. Além do mais, esses planos de cuidados propiciam diretrizes para documentação e instrumentos para a análise da assistência. (DOENGES, 2010)

A realização dos diagnósticos de enfermagem para o DM deve ser embasada no planejamento do cuidado prestado a esses portadores, contribuindo para aquisição e manutenção do autocuidado facilitando a sua aceitação ao tratamento, melhoria na qualidade da assistência, além de colaborar para o desenvolvimento do conhecimento, autonomia e habilidade no autocuidado do paciente $\mathrm{e}$ familiares (ANDRADE; JUNIOR; FILHO, 2012).

Sob essa ótica, observa-se a importância que o profissional enfermeiro possui no cuidado ao paciente diabético, este por sua vez, vai desde a orientação e educação dos pacientes, acompanhamento na evolução patológica até o controle das decorrentes complicações da doença, tornando o trabalho imprescindível à prática profissional e a constituição de uma eficaz rotina a ser problematizada pela reflexão. 
Objetivou-se refletir, sob a ótica discente, a atuação do enfermeiro no tratamento do $D i$ abetes Mellitus (DM) a partir da vivência em um Hospital Universitário do Interior nordestino.

\section{MÉTODOS}

Estudo de caráter empírico-descritivo, com enfoque exploratório, de relato de experiência vivenciado por discentes do curso de enfermagem da Universidade Federal de Campina Grande, do décimo período do curso de graduação, elaborado a partir das práticas do estágio curricular Supervisionado II, com carga horária de 420 horas.

O relato de experiência propõe tornar visível e compartilhar com outros profissionais e estudantes uma vivência prática, funcionando como um instrumento de pesquisa descritiva que proporciona uma reflexão sobre uma ação ou um conjunto de ações vivenciadas (SANTOS; ALMEIDA; REIS, 2013).

Ações desenvolvidas no Estágio Curricular Supervisionado II, caracterizado como uma modalidade de ensino obrigatória no Curso de Graduação em Enfermagem, que tem o propósito de propiciar ao acadêmico do último semestre do curso, uma visão de sua profissão de forma ampla e concreta. Apresenta como objetivo geral: Desenvolver habilidade técnicocientífica para atuar em serviços de saúde integrando os aspectos: planejamento, execução, supervisão, treinamento e avaliação das atividades de enfermagem, tomando como referencial teórico os princípios do Sistema
Único de Saúde (SUS), a Lei do Exercício Profissional. (PPC, 2011).

A experiência sucedeu na Ala $\mathrm{C}$ do Hospital Universitário Alcides Carneiro, em Julho de 2016. Durante esse período, buscou-se pontuar o caminho pelas estudantes percorrido, desafios enfrentados e experiência sucedida na Ala C, composta por 20 leitos, subdivididos em 6 enfermarias e de assistência voltada para a clínica feminina, com a maior predominância de pacientes endócrinos e cardíacos acompanhados por uma equipe multidisciplinar do Hospital Universitário Alcides Carneiro - HUAC.

O delineamento desta experiência se dá a partir das concepções formuladas por Holliday (2006) quando declara 5 etapas que norteiam a sistematização de experiências:

1) $O$ ponto de partida: a experiência construída a partir das reflexões da prática na ala $\mathrm{C}$;

2) As perguntas iniciais: Como refletir a prática do enfermeiro no cuidado ao cliente com DM? Que contribuições à atuação do enfermeiro no cuidado ao cliente pode trazer a qualidade da assistência?

3) Recuperação do processo vivido: Reconstrução do processo vivenciado a partir do pensamento crítico reflexivo;

4) A reflexão de fundo: descrição sintética do processo vivido.

5) Os pontos de chegada: principais conclusões a partir do aprendizado sobre a experiência vivida. 


\section{RESULTADOS E DISCUSSÃO}

Narrativa discente: a possibilidade de uma reflexão

Iniciamos o estágio em um Hospital Universitário localizado no estado da Paraíba em uma cidade do interior, no mês de Julho de 2016, fomos lotadas para a ala $\mathrm{C}$ por meio de sorteio, esta ala atende pacientes do sexo feminino, possui uma demanda significativa de pacientes majoritariamente portadores de diabetes melittus tipo II, boa parte vítima de amputação de membros inferiores, onde participamos do processo de trabalho em enfermagem. Nossa primeira competência foi realizar o diagnóstico situacional do ambiente em que estaríamos atuando.

A ala apresentava uma média de 18 pacientes ocupando leitos, uma media de 3 altas por dia e a mesma de admissão. Por se tratar de uma ala clinica, a procura por vagas em leitos era constante, o que justifica a constante ocupação dos leitos nas enfermarias. A equipe de enfermagem é composta pela enfermeira assistencial (plantonista), a enfermeira que tratava de assuntos puramente burocráticos (diarista) e a equipe de técnicos de enfermagem, uma média de quatro por plantão.

O diagnóstico situacional da Ala $\mathrm{C}$, nos permitiu identificar que a predominância da ala é de pacientes da clinica endócrina, pessoas idosas, em destaque por diagnostico de Diabetes Melittus, muitas com complicações em membros inferiores, que resultaram em amputações.

A ocorrência do controle inadequado, do tempo de evolução e de fatores genéticos da doença, resulta nas principais complicações do DM. A nefropatia diabética, a retinopatia diabética e a neuropatia diabética englobam as complicações crônicas microvasculares. Já as alterações nos grandes vasos como infarto agudo do miocárdio, acidente vascular cerebral e doença vascular periférica englobam as complicações crônicas macrovasculares (TSCHIEDEL,2014).

Os sinais e sintomas da neuropatia diabética podem variar de acordo como tipo de fibra que pode está envolvida, essa neuropatia sensitivo-motora pode desenvolver transtornos tróficos da pele e osteoarticulares dos membros inferiores como, por exemplo: atrofia muscular, redução da amplitude do movimento e, por conseguinte aumento do risco de ulceração plantar que poderá resultar em uma futura amputação. (BARRILE. et al, 2013).

Após esse diagnóstico desempenhamos atividades diárias no serviço, durante 60 turnos, seguíamos a rotina estabelecida pelos profissionais da ala, contribuíamos com o pensamento critico e reflexivo que a graduação nos proporcionou, refletimos e participamos ativamente do processo de trabalho da alta complexidade assim como também seus nós críticos.

Dentre as atividades realizadas pelo enfermeiro do setor, no cuidado ao paciente clinico, destacamos as seguintes: prestar cuidados que exijam uma maior habilidade técnica e raciocínio crítico, curativos de grande complexidade, sondagem vesical, gástrica e enteral, coletas de gasometrias, eletrocardiogramas, evoluções de enfermagem, 
burocracias de exames, controle de antibióticos, supervisão da equipe de enfermagem.

Durante a vivência, foi imprescindível a inserção dos discentes nas atividades da ALA C, os quais tiveram a oportunidade de participar de forma diária das competências do enfermeiro juntamente com a participação da equipe multidisciplinar.

Algumas dificuldades foram identificadas como, o déficit de insumos, a não interação profissional-profissional, profissionalcoletividade.

O estágio supervisionado é um momento impar na formação do discente, contribui na solidificação do saber e o prepara para a vida profissional.

\section{CONCLUSÃO}

O estágio curricular supervisionado é considerado um componente fundamental no ensino superior, pois demonstra a relação entre as intenções de formação e o campo social e profissional, além de constituir como prática investigativa,

É uma etapa extremamente expressiva no processo de formação do aluno, no qual proporciona vivenciar a rotina hospitalar por um período prolongado, como também é uma alternativa fundamental para que haja uma familiaridade maior com as atribuições do profissional de enfermagem do nível superior.

Atuar na ALA C foi extremamente importante no processo de desenvolvimento e aprendizagem, pois promoveu oportunidades de vivenciar na prática conteúdos acadêmicos, propiciando desta forma, a aquisição de conhecimentos e atitudes relacionadas com a profissão. Permitiu ainda a troca de experiências entre a equipe (técnicos de enfermagem, enfermeiros, médicos, auxiliares de serviços gerais) e familiares dos pacientes, bem como o intercâmbio de novas ideias, conceitos, planos e estratégias.

Portanto, acredita-se que esta narrativa seja um produto que possa contribuir para reflexões sobre rotinas em alas de hospitais, como também a necessidade do processo de trabalho em saúde como fundamental à prática de enfermagem e, consequentemente, ao cuidado.

\section{REFERÊNCIAS}

ANDRADE, Joyce Paloma Xavier; JUNIOR, José Vasconcelos de Carvalho; FILHO, Wellington $\mathrm{Mu}-$ carbel dos Santos. Principais diagnósticos de enfermagem da NANDA para portadores de diabetes tipo II nas equipes de saúde da família do município de Arcoverde - PE. Saúde Coletiva em Debate. [S. 1]: v. 2, n. 1, p. 1-8, dez. 2012. Disponível em http://fis.edu.br/revistaenfermagem/artigos/vol02/artig o01.pdf acesso em 08 Out, 2016.

BARRILE, S. R. et al. Comprometimento sensóriomotor dos membros inferiores em diabéticos do tipo 2 . Fisioter Mov. p. 537-548. Curitiba, jul/set 2013. Disponível em http://www.scielo.br/pdf/fm/v26n3/a07v26n3.pdf acesso em 25 Ago,2016.

BRASIL, Lei 7.498, de 25 de junho de 1986. Dispõe sobre a Regulamentação do Exercício da Enfermagem e dá outras providências. Brasília: Ministério da Saúde; 1986. Disponível em:<http://www2.camara.gov.br/internet/legislacao/le gin.htm>. Acesso em 20 Ago. 2016.

DOENGES, M. E. MOORHOUSE, M. F. MURR, A. C. Diagnósticos de enfermagem: intervenções, prioridades e fundamentos; tradução Carlos Henrique Cosenday; revisão técnica Sonia Regina de Souza. Rio de Janeiro: Guanabara Koogan, 2010. 
DIRETRIZES DA SOCIEDADE BRASILEIRA DE DIABETES: 2013-2014/Sociedade Brasileira de Diabetes; [organização José Egidio Paulo de Oliveira, Sérgio Vencio]. - São Paulo: AC Farmacêutica, 2014. Disponível em

http://www.sgc.goias.gov.br/upload/arquivos/201405/diretrizes-sbd-2014.pdf acesso em 07 Out, 2016.

MENICUCCI, T. M. G.. História da reforma sanitária brasileira e do Sistema Único de Saúde: mudanças, continuidades e a agenda atual. História, Ciências, Saúde - Manguinhos, Rio de Janeiro, v.21, n.1, jan.mar. 2014, p.77-92. Disponível em http://www.scielo.br/pdf/hcsm/v21n1/0104-5970hcsm-21-1-00077.pdf acesso em 20 Set, 2016

Ministério da Saúde. Secretaria de Atenção à Saúde. Departamento de Atenção Básica. Diabetes Mellitus / Ministério da Saúde, Secretaria de Atenção à Saúde, Departamento de Atenção Básica. Brasília: Ministério da Saúde, 2006. 64 p. il. - (Cadernos de Atenção Básica, n. 16) (Série A. Normas e Manuais Técnicos). Disponível em http://bvsms.saude.gov.br/bvs/publicacoes/estrategias_ cuidado_pessoa_diabetes_mellitus_cab36.pdf Acesso em: 06 Set, 2016.

Projeto pedagógico do curso de enfermagem do CCBS-UFCG. CCBS: UFCG, 2011.

TOSCANO, C.M. As campanhas nacionais para a detecção das doenças crônicas não-transmissíveis: diabetes e hipertensão. Revista Ciência \& Saúde Coletiva. p. 885-895, Out 2004. Disponível em http://www.scielo.br/scielo.php?pid=S1413$81232004000400010 \&$ script $=$ sci_abstract $\&$ tlng $=$ pt acesso em 26 Set, 2016.

TSCHIEDEL, B. Complicações crônicas do diabetes. Endocrinologia. p. 7-12. Set/out 2014. Disponível em http://files.bvs.br/upload/S/0047-

2077/2014/v102n5/a4502.pdf acesso em 15 Set,2016.

TEIXEIRA, Carla Regina de Souza et. al. Validação de intervenções de enfermagem em pessoas com diabetes mellitus. Revista Esc. De Enfermagem. São Paulo: v. 45, n. 1, p. 173-179, 2011. Disponível em http://www.scielo.br/scielo.php?script=sci_arttext\&pi $\mathrm{d}=$ S0080-62342011000100024 acesso em 01 Out, 2016.

\section{Dandara Medeiros Paiva}

Graduada pela Universidade Federal de Campina Grande-UFCG.

\section{Joseane Xavier de Almeida \\ Preceptora do estágio supervisionado II. \\ Graduada pela União de Ensino Superior de Campina Grande-UNESC.}

\section{Laura Raquel Araújo de Moraes \\ Especialista em auditoria em enfermagem. \\ Graduada pela Universidade Federal de Campina \\ Grande-UFCG.}

\section{Maria Érica Gomes da Silva}

Enfermeira Residente em Obstetrícia, no Hospital Dom Malan IMIP-PE. Graduada pela Universidade Federal de Campina Grande-UFCG.

Rosangela Vidal de Negreiros
Mestre em enfermagem, docente do Curso de
graduação em enfermagem do Centro de Ciências
Biológicas e da Saúde-CCBS da Universidade
Federal de Campina Grande-UFCG.

\section{Rosangela Vidal de Negreiros}

graduação em enfermagem do Centro de Ciências

Federal de Campina Grande -UFCG.

\footnotetext{
Joanna Renata Santos de Almeida Silva

Graduada pela Universidade Federal de Campina Grande-UFCG.
} 